\title{
Recovery of glutamic acid from ultrafiltration concentrate using diafiltration with isoelectric supernatants
}

\author{
Guojun Zhang*, Zhongzhou Liu ${ }^{\mathrm{a}}$, Liang Zhao ${ }^{\mathrm{a}}$, Hongbing $\mathrm{Li}^{\mathrm{a}}$, Quan Zhou ${ }^{\mathrm{a}}$, \\ Fei He ${ }^{\mathrm{a}}$, Zhaohui $\mathrm{Xu}^{\mathrm{b}}$, Huanzhang Wang ${ }^{\mathrm{b}}$ \\ "Research Center for Eco-Environmental Sciences, Chinese Academy of Sciences, \\ P.O. Box 287l, Beijing, 100085, P.R. China \\ Tel.+86 (10) 6284 9195; Fax+86 (10)6292 3441; email: zhangguojunmh@yahoo.com \\ ${ }^{h}$ Star Lake Co. Inc. Zhaoqing Guangdong, Zhaoqing, 526060. P.R. China
}

Received 10 October 2001; accepted 12 June 2002

\begin{abstract}
A new diafiltration method for recovery of glutamic acid (GA) from ultrafiltration concentrate by continuously feeding isoelectric supernatants was developed. A developed model of GA recovery ratio was used to simulate the process of diafiltration with isoelectric supernatants, which demonstrated higher recovery ratio and water saving than the traditional dilfiltration with water. Coupled with the technique of distillation and isoelectric crystallization, the GA purity and yicld were increased by $9.3 \%$ and $3.35 \%$ respectively. Then, a novel flow sheet of GA isolation from fermentation broth was formed.

Keywords: Glutamic acid; Ultrafiltration concentrate; Diafiltration; Isoelectric supernatants; Distillation; Isoelectric crystallization

1. Introduction

L-glutamic acid (GA), in the form of monosodium salt, is widely used as a seasoning throughout the world. Nowadays, the production of monosodium salt in China is higher than any other country in the world. The total Chinese production of monosodium glutamate in 1998 exceeded

\footnotetext{
*Corresponding author.
}

590,300 tons [1]. Traditionally, GA is isolated from fermentation broth by isoelectric crystallization with or without prior removal of biomass. However, the presence of biomass would reduce the yield of crystallization, favoring formation of $\beta$-form crystals, especially at higher concentrations [2]. Ultrafiltration (UF) process has been widely used for concentration, separation and purification. An attractive feature of UF is its simplicity in
\end{abstract}


operation and does not require a phase change. Ultrafiltration and diafiltration (DF) have been used to concentrate and purify many types of products in different fields. One of the drawbacks associated with UF is that the recovery of some useful substances contained in UF concentrate is limited. Kuo and Chiang [3] used ultrafiltration to remove bacterial from $\mathrm{GA}$ fermentation broth. The retentivity of the UF membrane with glutamic acid was practically zero, thus the cell-free glutamic acid could be recovered in the UF permeate. The higher the volume concentration ratio (VCR), the higher recovery for glutamic acid could be accomplished by UF. Although the recovery ratio increased with increasing VCR, the low permeate flux at high VCR may entail processing difficulties. Therefore, DF process mode should be used at a certain VCR of UF process to "wash out" the remaining GA in the UF concentrate, thus enhancing GA recovery.

DF is simply an alternative method of operating an ultrafilter where a solvent, typically water, is combincd with the feed to the ultrafilter. This has the effect of conveniently "washing out" any substances not retained by the membrane [4]. DF is a very versatile operation and is not only limited to water. Any compatible solvent will work just as well. In fact, DF can be used to transfer GA from UF concentrate to UF permeate. The constant volume batch diafiltration mode was used, in which the diafiltratering water was added to the feed tank at a rate equal to UF permeate flux to maintain a constant volume [3]. The additive in DF process conventionally was water, and no GA was introduced into the UF unit. The recovery of GA was always less than $100 \%$ due to the residual GA in ultrafiltration concentrate. In addition, much water was needed in order to obtain a certain recovery ratio and thus the total permeate volumes were increased in UF and DF processes. About $1.0-2.0 \%$ residual $\mathrm{GA}$, usually contained in isoelectric supernatants, needed to be put into ion exchange resins to recover the residual GA and then discharged, which caused the loss of GA. In this work, UF was initially used to clarify the broth. After the distillation and crystallization of UF permeate, some parts of the isoelectric supernatants were continuously recycled to wash out the residual $\mathrm{G} \Lambda$ in the UF concentrate during diafiltration process, which reduced water consumption in DF process and the burden of resins subsequently. The purpose of this work was to enhance the recovery of glumatic acid while reducing the total permeate volumes in UF and DF processes. This novel technology process is favorable for GA production.

\section{DF mathematical model of recovery fraction}

The system employed in the experimental tests was modeled as continuous stirred tank reactor (CSTR) whose flow sheet was shown in Fig. 1. Some experiments proved perfect agreement between the experimental response to a step variation and the one calculated by a CSTR model.

In the case of washing with water, mass balances yield the following expression for the component concentration in the UF permeate [5].

$$
C_{V_{D}}=C_{0}(1-\sigma) e^{-(1-\sigma) V_{D}}
$$

where $V_{D}$ is volumes permeate/volume of solution to be treated, $V_{D}$ can be defined as:

$$
V_{D}=\frac{V_{P D}}{V_{0}}
$$

$V_{p D}$ is volume of permeate in the diafiltration process; $V_{0}$ - initial volume of solution to be treated;

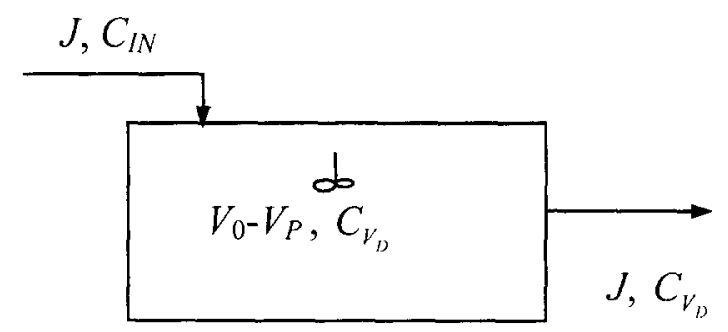

Fig. 1. Simple DF mathematical model, CSTR. 
$C_{1, \nu}$ - component concentration in the permeate at $V_{D}$ time; $C_{0}-$ initial component concentration in the solution to be treated; $\sigma$-retentate coefficient of the component.

In the following, the equations for the recovery ratio model are presented. In this work, $n$ is given by:

$$
n=\frac{V_{0}}{V_{0}-V_{P}}
$$

where $n$ is the volume concentration ratio (VCR) in UF process; $V_{0}$ - initial volumes of solution to be treated; $V_{p}$--volume of liquid permeated (l) in UF process without DF.

An alternative way of investigating ultrafiltration is to formulate the flux in terms of the transmembrane pressure driving force [6]. This is the osmotic pressure approach in which the flux is given by:

$$
J=\frac{\Delta P-\pi}{\mu_{s} R_{m}}
$$

where $\Delta P$ is transmembrane pressure; $\pi$-osmosis pressure in the UF concentrate; $\mu_{s}$ - solution viscosity $\left(\mathrm{N} . \mathrm{s} / \mathrm{m}^{2}\right) ; R_{m}-$ membrane resistance $\left(\mathrm{m}^{-1}\right)$, $\pi$ is the function of salt concentration. In the case of washing with water, UF flux will increase due to osmosis pressure changes with salt concentration changing. But in this work, in the case of washing with isoelectric supernatants, osmosis pressure would be approximately constant due to salt balance between the additive and the permeatc. Consequently it is assumed that UF flux is not changed in the DF process.

In the case of washing with a solution containing a component, a mathematical model has to be developed for the component concentration in the retentate. Barba and Beolchini [7] have developed the following expression for the component concentration in the permeate:

$$
C_{V_{s}}=C_{I N}-\left[C_{I N}-C_{0}(1-\sigma)\right] e^{-(1-\sigma) r_{t \prime}}
$$

where $C_{0}$ is initial component concentration in the solution to be treated; $C_{I N}$ - component concentration in the washing solution; $\sigma$ - retentate coefficient of the component; $V_{D}$-volumes permeate/ volume of solution to be treated.

For small changes in the liquid permeate, $V_{D}$, the change in the total recovery ratio of component in the system, expressed as $\eta$, is:

$\eta_{t}=1-\frac{1}{n}+\frac{\int^{p_{D}} \mathrm{~d} V_{P D}}{V_{0} C_{0}}$

where $1-1 / n$ is the recovery ratio of the component in UF without DF; $\int_{0}{ }^{\prime}{ }^{\prime} C_{i b} \mathrm{~d} V_{P D} / V_{0} C_{0}$ the recovery ratio of the component when the washing solution, $V_{D}$ was introduced to UF concentrate.

Substituting Eqs. (2) and (5) to Eq. (6) and integrating from $0-V_{D}$ at limiting flux, recovery ratio can be calculated by the following equation:

$$
\begin{aligned}
& \eta_{\imath}=1-\frac{1}{n}+\frac{C_{I N} V_{D}}{C_{0}} \\
& +\frac{C_{I N}-C_{0}(1-\sigma)}{C_{0}(1-\sigma)}\left(e^{-(1-\sigma) V_{D}}-1\right)
\end{aligned}
$$

It is essential to point out that Eq. (7) is only suitable to UF-DF unit but not to the whole process. Because the residual GA from isoelectric supernatants was introduced into UF unit, 100\% recovery could be reached only for UF-DF unit. But for the whole process including the subsequent process of crystallization, GA recovery ratio was still less than $100 \%$ based on mass balances since there was always a little residual GA discharged.

\section{Experiments}

\subsection{Flow sheet}

The conventional and novel experimental technological processes were shown in Figs. 2a, $\mathrm{b}$ and $\mathrm{c}$. 

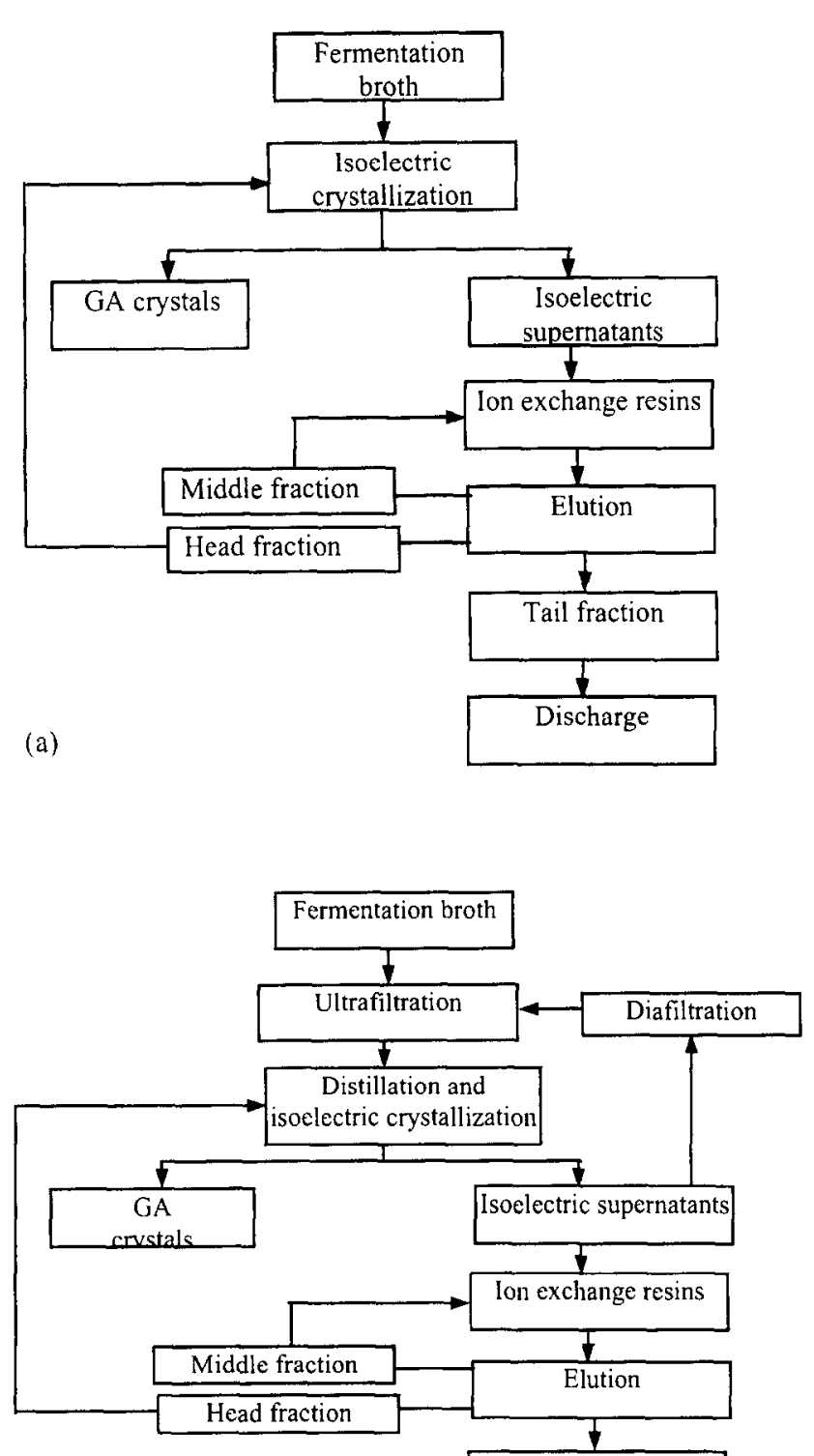

(c)

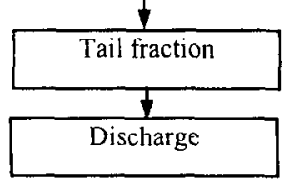

The conventional process of GA isolation from fermentation broth, as shown in Fig. $2 \mathrm{a}$, is isoelectric crystallization without prior removal of biomass. GA recovery in Fig. $2 \mathrm{a}$ is less than that in Fig. $2 b$ and $2 c$ due to the effect of biomass on crystallization. Fig. $2 \mathrm{~b}$ is the conventional process

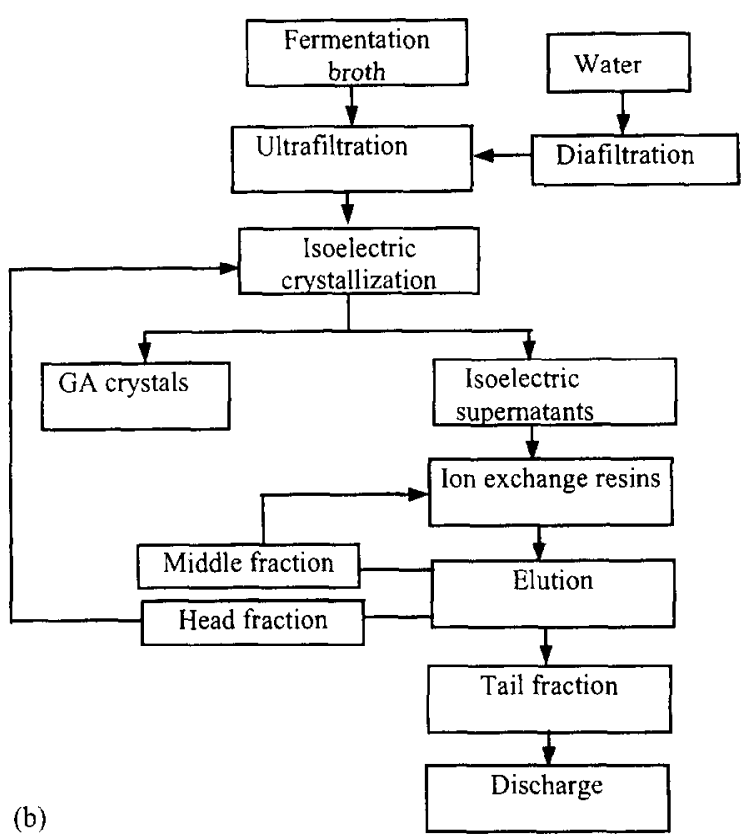

Fig. 2. Comparison of technological processes; a, conventional technological process without UF and DF; b, technological process including UF and DF with water; $\mathrm{c}$, novel technological process including UF and DF with isoelectric supernatants.

of prior removal of biomass using UF and DF, which was investigated by KUO and others [2]. In this process, much water was needed to diafiltrate the GA of UF concentrates in order to obtain higher recovery ratio. Fig. $2 \mathrm{c}$ was the novel flow shect in this work. Compared with Fig. 2b, 
the supernatants were recycled as diafiltrating solution, which saved a great amount of water, and the crystallization was also different from conventional method, which enhanced the purity and yield of product. In addition, the burden of ion exchange resins would be lightened since some parts of the isoelectric supernatants were recycled as diafiltrating solution but not be put into resins.

\subsection{Fermentation broth}

The GA fermentation broth, supplied by Starlake Co. (ZhaoQing, GuangDong province), was produced by cultivating corynebacterium crenatum with hydrolytic sugar, corn steep liquor, urea, minerals, etc., as nutrients and polyoxalkylene glycerol ether as the antifoaming agent. The typical constituents of the broth are listed in Table 1.

Table 1

Some primary physical and chemical properties of the glumatic acid fermentation broth

\begin{tabular}{ll}
\hline $\mathrm{GA}, \%$, w/v & $9.0-10.5$ \\
$\mathrm{pH}$ & $6.4-6.9$ \\
Wet basis, \%, w/v & $3.5-4.5$ \\
Residual sugar, \%, w/v & $0.5-0.75$ \\
\hline
\end{tabular}

\subsection{Ultrafiltration and diafiltration}

The experimental set-up is shown in Fig. 3. The polyvinylidene fluoride (PVDF) and polyacrylic nitrile (PAN) membranes, made in our workshop were employed in this experiment. The molecular weight cut-off ( $\mathrm{MWCO}$ ) of the membranes was 60,000 and the effective membrane area was $1 \mathrm{~m}^{2}$. The hollow fiber UF modules were cross-flow system. Rotameters were used to measure flow rates. A continuous agitation and temperature control was provided for the feed vessel. The feed solution at a given flow rate was supplied to the membrane module by means of centrifugal feed pump, the concentrate was recirculated to the feed vessel while permeate was collected in the permeate tank. Initial feed volumes was recorded and target permeate volume was calculated by substituting target VCR value to Eq. (3). When the UF permeate volumes reached a target value i.e., a target VCR value, water or isoelectric supernatants in DF process were added simultaneously to the beaker under agitation through a peristaltic pump.

\subsection{Crystallization}

\subsubsection{Distillation and isoelectric crystallization}

To simulate the industrial conditions, the UF and DF permeate was introduced into a 101

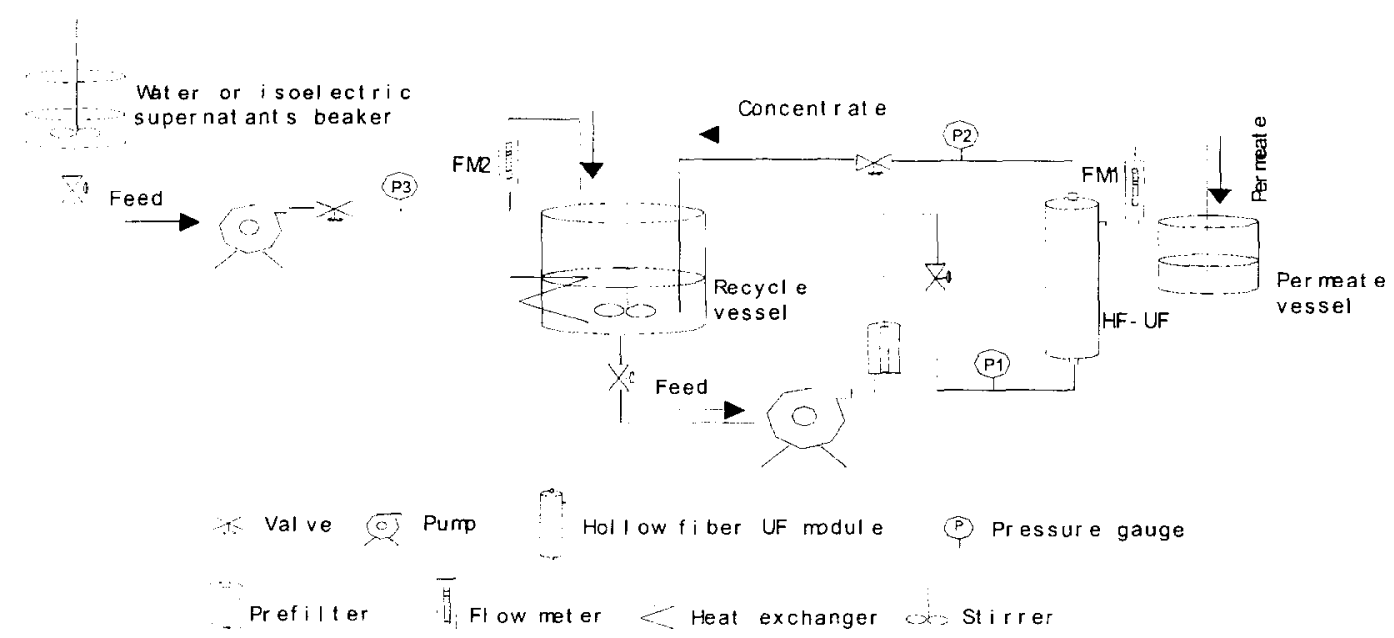

Fig. 3. Experimental apparatus employed for ultrafiltration and diafiltration. 
beaker. Sulphuric acid was slowly added to the permeate at $30^{\circ} \mathrm{C}$ under constant agitation until the $\mathrm{pH}$ reached 4.5 , then crystal seeds were added to the UF permeate which was concentrated by distillation to approximately 2.5-fold. Again sulphuric acid was slowly added to the solutions until the $\mathrm{pH}$ reached 3.0-3.2, the isoelectric point of glumatic acid. Thereafter, the solutions were standing for $1.5 \mathrm{~h}$ at $5^{\circ} \mathrm{C}$ to precipitate the crystals. Supernatants were taken from the beaker to diafiltrate UF concentrate, enhancing GA recovery.

\subsubsection{Conventional crystallization}

The conventional method of crystallization was also carried out as a contrast. Sulphuric acid was slowly added to the fermentation broth at $5^{\circ} \mathrm{C}$ under agitation at $200 \mathrm{rev} \mathrm{min}^{-1}$ until the $\mathrm{pH}$ reached 3.0-3.2 for $5 \mathrm{~h}$ and the solution left standing for another $1-1.5 \mathrm{~h}$.

\subsection{Analytical methods}

GA in the fermentation broth and isoelectric supernatants was quantified by measuring the carbon dioxide evolved through enzymic decarboxylation with a Warburg apparatus. For crystallized glutamic acid, purity was measured by polarimetric method and transmittance by spectrophotometric method at $590 \mathrm{~nm}$ in $2 \mathrm{~mol} . \mathrm{dm}^{-3}$ hydrochloric acid solutions with $1 \mathrm{~cm}$ optical path. Residual sugar was analyzed by Fehling's method [8].

\subsection{Sample preparation of isoelectric supernatants}

The UF permeate was introduced into a 101 beaker. After distillation and isoelectric crystallization, the GA crystals were filtered and the isoelectric supernatants were reserved. The composition of isoelectric supernatants was analyzed according to analytical methods above-mentioned. The GA concentration is about $2 \%$ and $\mathrm{pH}$ is about 3.1-3.2. Half of the isoelectric supernatants were diluted to $1 \%$ with water in order to investigate the effect of isoelectric supernatant concentration on GA recovery ratio in DF process.
3.7. Calculation methods of experimental recovery ratio

Experimental recovery ratio of GA, expressed as $\eta_{e}$, was calculated by comparing the pure GA quality before and after UF-DF process as follows:

$\eta_{e}=\frac{V_{P} C_{0}+V_{P D} C_{P D}}{V_{0} C_{0}}$

\section{Results and discussion}

\subsection{UF performance}

As UF proceeded, the biomass concentration, $C$ increased and flux rate, $J$ decreased, as shown in Figs. $4 \mathrm{a}$ and $4 \mathrm{~b}$. A plateau occurred on the curve, beyond this, the gel polarization model held and flux declined with $\ln C$ [2]. In other conditions, such as higher biomass concentration or lower circulation velocity, flux rate declined logarithmically from the beginning with no plateau region (data not shown).

In UF experiments, the average flux can be calculated by the following expression:

$$
V_{a v}=\frac{V_{\text {total }}}{t}
$$

where $V_{a l}$ is average flux of different modules; $V_{\text {total }}$ - total permeate volumes in one period; $t$ operation time in one period.

As shown in Fig. 5, the average fluxes of two kinds of membrane were compared. Some literature $[9,10]$ have reported that hydrophilic membrane is more antifouling than hydrophobic membrane especially in the process of treating protein solution. In our experiments this conclusion was validated. Fig. 4 showed that the average flux (in situ UF experiments, about $5 \mathrm{~h}$ as an operation period) of PAN membrane was higher than that of PVDF membrane. Therefore, PAN UF membrane was employed in the DF experiments. 

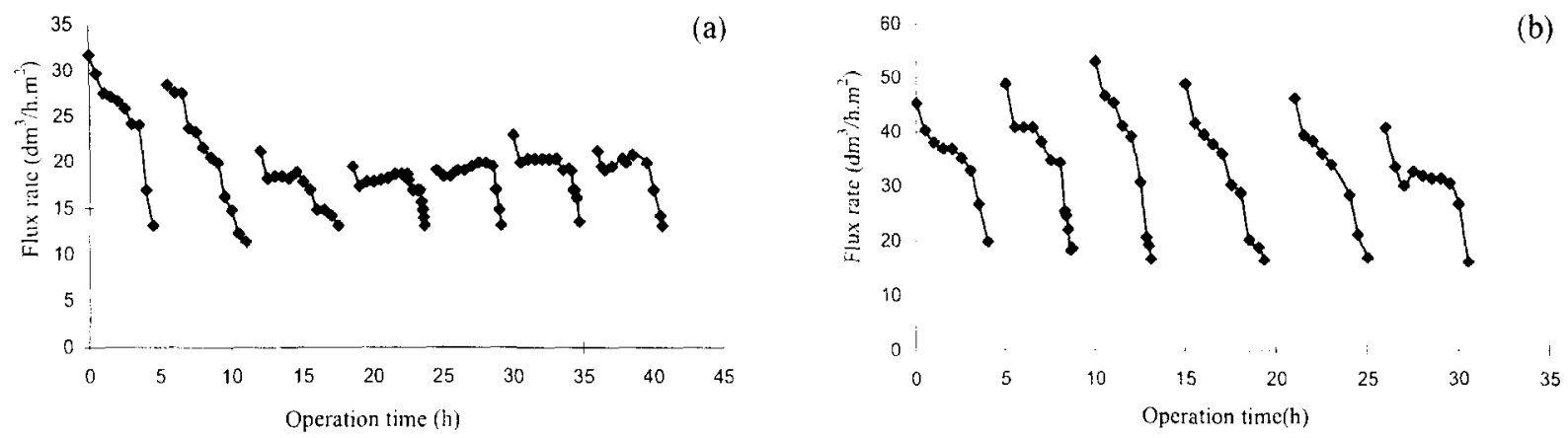

Fig. 4. Membrane flux rate changes with operation time in UF process; a, PVDF membrane flux rate changes with operation time; b, PAN membrane flux rate changes with operation time; $P_{I}=2.6 \mathrm{MPa} ; P_{0}=2.0 \mathrm{MPa} ; P_{1}-$ retentate inlet pressure; $P_{0}$ - retentate outlet pressure; fermentation broth $100-120 \mathrm{dm}^{3} ;$ GA $9.0-10.5 \%$, w/v; wet biomass concentration $3.5-4.5 \%$, w/v; about $5 \mathrm{~h}$ as one operation period. After each period, cleaning with sodium hydroxide was carried out under $0.1 \mathrm{MPa}$ pressure.

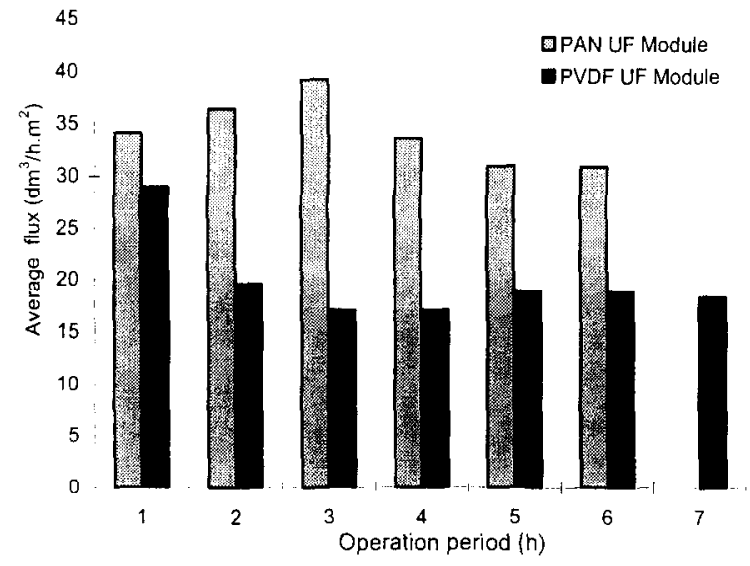

Fig. 5. Average flux of different membrane module; $P_{1}=2.6 \mathrm{MPa} ; P_{0}=2.0 \mathrm{MPa} ; P_{1}-$ retentate inlet pressure; $P_{1}$ - retentate outlet pressure; fermentation broth 100 $120 \mathrm{dm}^{3}$; GA $9.0-10.5 \%$, w/v; wet biomass concentration $3.5-4.5 \%, \mathrm{w} / \mathrm{v}$; about $5 \mathrm{~h}$ as one operation period.

\subsection{DF performance}

Continuous diafiltration involves adding washing solution at the appropriate $\mathrm{pH}$ and temperature to the feed tank at the same rate as the permeate flux, thus keeping the feed volume $\left(V_{0}-V_{p}\right)$ constant during the process. In this work, in order to meet with the company's request, which included the total permeate volumes in UF and DF processes must be no more than 1.2-fold initial solution volumes and the recovery ratio of GA must be more than $98.5 \%$, a 10 -fold concentration of initial solution to be treated was chosen as the point to end UF and start DF. When the diafiltration volumes reached a target value, the operation was stopped. In Fig. 7, when diafiltrating solution is water, VCR is 10 and $V_{D}$ is 0.3 , the total permeate in UF-DF process is 1.2-fold initial solution volumes and the recovery ratio is less than $98.5 \%$ which cannot meet with the company's request. Therefore, a new diafiltration method using isoelectric supernatant to diafiltrate UF concentrate was proposed. Obviously, in the case of a washing solution containing $\mathrm{GA}$ at a certain concentration, as shown by Figs. 6 and 7, the higher GA concentration and recovery ratio of UF-DF process can be certainly reached with adding isoelectric supernatants rather than that with adding water in the same DF volumes. So, when obtaining the same recovery ratio, DF with isoelectric supernatants can reduce the total volumes of UF and DF processes.

Some continuous diafiltration tests have been performed in order to validate Eq. (7). The continuous lines have been calculated by Eq. (7) for washing with isoelectric supernatants (1\% and $2 \%$ ). The GA retentate coefficient was experimentally estimated according to the literature [7] 


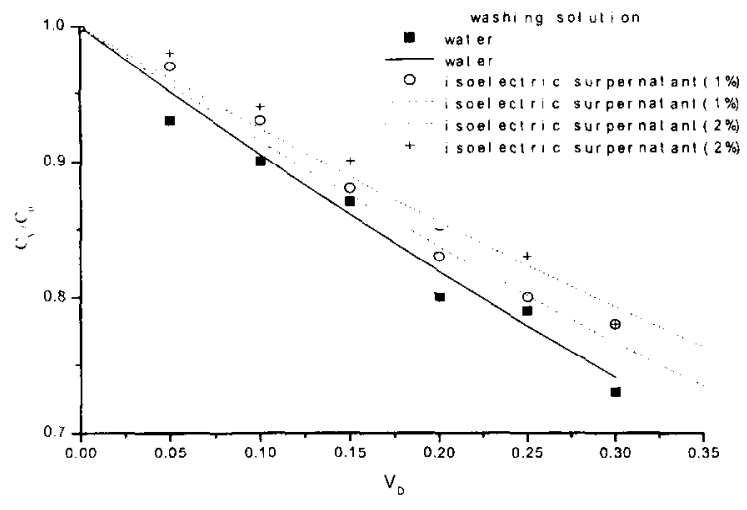

Fig. 6. Experimental, points, and predicated, lines, dimensionless GA concentration vs. diluted volumes profiles during diafiltration of a GA solution of $10 \%$ using distilled water and isoelectric supernatants, $1 \%$ and $2 \%$, as washing solutions. Lines have been calculated by Eqs. (1) and (5).

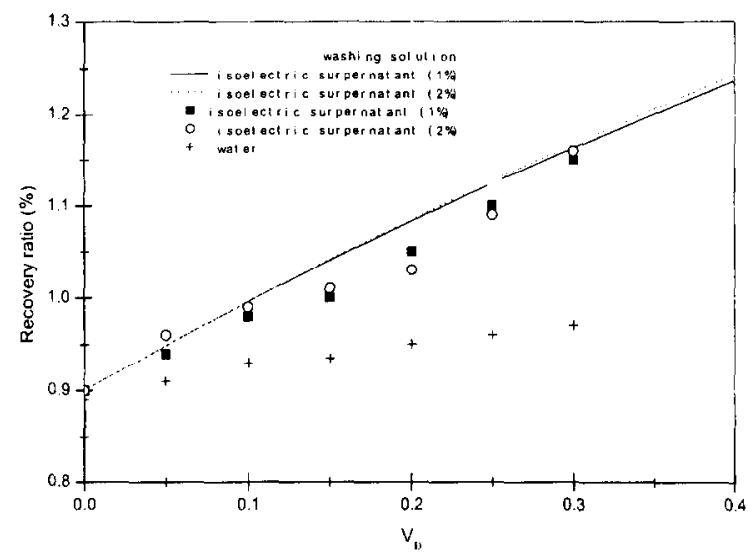

Fig. 7. Experimental, points, and predicated, lines, dimensionless GA concentration vs. diluted volumes profiles during diafiltration of a GA solution of $10 \%$ using distilled water and isoelectric supernatants, $1 \%$ and $2 \%$, as washing solutions. Lines have been calculated by Eq. (7); points have been calculated by Eq. (8).

at 0 for a pure GA. There is good agreement between the experimental (dots) and calculated (lines) data in both tests. In particular, the curves obtained with water and isoelectric supernatants as washing solution validate the modified model.

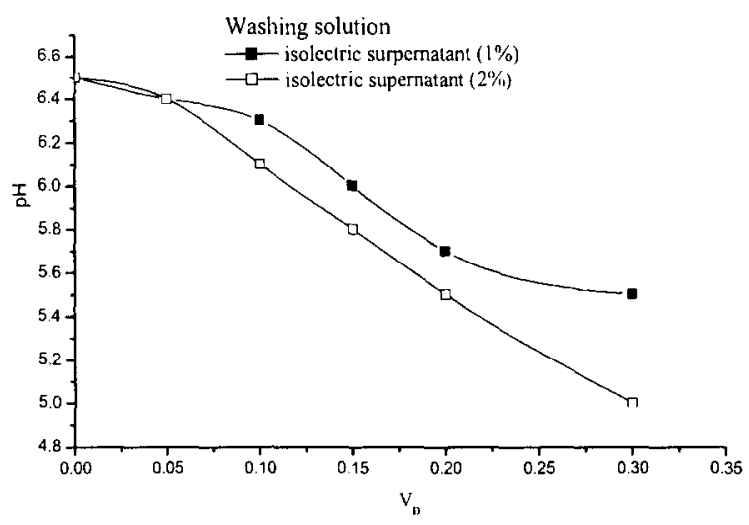

Fig. 8. $\mathrm{pH}$ changes with $\mathrm{DF}$ volume, $V_{D}$ changing.

It should be pointed out that the relationships between $C_{V D}, \eta$, and $V_{D}$ are curvilinear in according to Eqs. (1),(5) and (7), but because $V_{D}$ value is relatively low, the figures only show part of the straight line.

As shown in Fig. 8, because isoelectric supernatants, in the form of tiny trickles, were added to UF concentrate in the DF process, slight change of $\mathrm{pH}$ arose, but far from isoelectric point of GA. We cannot observe crystals precipitating in DF process and DF would also not influence the crystallization but reduce acid consumption in the subsequent crystallization process since the $\mathrm{pH}$ of isoelectric supernatants, which was added to UF concentrate, was about 3.1-3.2.

\subsection{Advantage of proposed crystallization over conventional method}

To simulate the industrial condition, the permeate in UF and DF processes was collected and then crystallized by the proposed method i.e. distillation and isoelectric crystallization. As a contrast, the conventional method was also carried out. Table 2 shows the results, indicating the proposed was superior in terms of product quality and yield. Thus, a novel flow sheet including UFDF, distillation and isoelectric crystallization was formed. 
Table 2

Comparison between present method and conventional crystallization

\begin{tabular}{llll}
\hline & \multicolumn{2}{l}{ Product quality } & Yields, \\
\cline { 2 - 3 } & $\begin{array}{l}\text { Purity, } \\
\%\end{array}$ & $\begin{array}{l}\text { Transmittance, } \\
\%\end{array}$ & \\
\hline $\begin{array}{l}\text { Present method } \\
\text { Conventional } \\
\text { method }\end{array}$ & 98.3 & 97.5 & 87.10 \\
\hline
\end{tabular}

\section{Conclusions}

A new method for recovery of glutamic acid from ultrafiltration concentrate was presented in this work. Experimental results have validated the developed model of GA recovery ratio for a particular component concentration in the retentate during diafiltration where washing is performed with isoelectric supernatants. Mass balances and the modificd mathematical model have allowed simulation of a diafiltration with isoelectric supernatants, which demonstrate higher recovery ratio and water saving with respect to a conventional dilfiltration with water. This aspect is very interesting considering that water costs are quite important in the overall operating costs of diafiltration process. Furthermore, and considering that high recovery ratio, the diafiltration process with isoelectric supernatants might be realized in industry.

In comparison with the conventional technological process, a novel flow sheet including UF-DF, distillation and isoelectric crystallization can increase the product purity and yield of GA by $9.3 \%$ and $3.35 \%$, respectively. The gains in GA would compensate for the additional cost of membrane system and the increased purity of GA would ease the subsequent process of conversion to the monosodium salt.

\section{Symbols}

$C_{0} \quad$ - Initial component concentration in the solution to be treated, $\mathrm{mg} / \mathrm{l}$
$C_{I N}$ - Component concentration in the washing solution, $\mathrm{mg} / \mathrm{l}$

$C_{I D}$ - Component concentration in the permeate at $V_{l}$ time

$J \quad$ - UF flux in DF process, $1 / \mathrm{m}^{2} \cdot \mathrm{h}$

$n$ - Volume concentration ratio, VCR, in UF process

$R_{m} \quad$ - Membrane resistance, $\mathrm{m}^{-1}$

$t^{m} \quad$ - Operation time in one period

$V \quad$ - Initial volume of solution to be treated

$V_{a r} \quad$ - Average flux of different modules

$V_{D}$ D - Volumes permeate/volume of solution to be treated

$V_{P l}$ - Volume of permeate in the diafiltration process

$V_{\text {total }}$ - Total permeate volumes in one period

\section{Greek}

$\Delta p \quad$ - Transmembrane pressure

$\eta_{\varepsilon} \quad$ - Experimental component recovery ratio, $\%$

$\eta$, Theoretical component recovery ratio, $\%$

$\mu_{s} \quad$ - Solution viscosity, $\mathrm{N} \mathrm{s} / \mathrm{m}^{2}$

$\pi$ - Osmosis pressure in the UF concentrate

$\sigma \quad$ - Retentate coefficient of the component

\section{Acknowledgements}

This work was supported by the Chinese Academy of Sciences, Project KZ951-A1-201-02, KZ95T-05. We would like to thank Dr. Yuansheng Pei for the preparation of this manuscript.

\section{References}

[1] H. Wang and L. Zhao, Application of membrane separation technology to treat wastewater of monosodium glutamate, Membr. Sci. Technol., 20(4) (2000) 62-64 (in Chinese).

[2] S. Huang, X. Wu, C. Yuan et al. [all authors?], Application of membrane filtration to glutamic acid recovery, J. Chem. Tech. Biotechnol., 64 (1995) 109114.

[3] W.S. Kuo and B.H. Chiang, Recovery of glutamic acid from fermentation broth by membranc processing, $\mathrm{J}$. 
Food Sci., 52 (1987) 1401-4.

[4] E. Bjurstrom, Biotechnology: fermentation and downstream processing, Chem. Eng., 92 (1985) 12658.

[5] M. Cheryan, Ultrafiltration Handbook, Technomic Publishing Co., Lancaster, PA., 1986, pp. 293-311.

[6] G. Foley and J. Garcia, Ultrafiltration flux theory based on viscosity and application to diafiltration optimization, J. Membr. Sci., 176 (2000) 55-61.

[7] D. Barba and F. Beolchini, Minimizing water use in diafiltration of whey protein concentrates, Separ. Sci.
Technol., 35(7) (2000) 951-965.

[8] Tianjin Institute of Light Industry, Analytical Methods in Fermentation Industry, Light Industry Publisher, Beijing, 1980, pp. 16-17.

[9] M. Nyström, Fouling of unmodified and modified polysulfone ultrafiltration membranes by ovalbumin, J. Membr. Sci., 44 (1989) 183-196.

[10] G. Capanneli, A. Bottino, V. Gekas et al. [all authors?] Protein fouling behavior of ultrafiltration membranes prepared with varying degrees of hydrophilicity, Process Biochem. Lut., 25(6) (1990) 221-224. 